\title{
Fiducial cross sections for Higgs boson production in association with a jet at next-to-next-to-leading order in QCD
}

\author{
Fabrizio Caola, ${ }^{1, *}$ Kirill Melnikov, ${ }^{2, \dagger}$ and Markus Schulze ${ }^{1, \$}$ \\ ${ }^{1}$ CERN Theory Division, CH-1211, Geneva 23, Switzerland \\ ${ }^{2}$ Institute for Theoretical Particle Physics, KIT, Karlsruhe D-76128, Germany
}

(Received 17 August 2015; published 21 October 2015)

\begin{abstract}
We extend the recent computation of Higgs boson production in association with a jet through next-tonext-to-leading order in perturbative QCD by including decays of the Higgs boson to electroweak vector bosons. This allows us to compute fiducial cross sections and kinematic distributions including realistic selection criteria for the Higgs boson decay products. As an illustration, we present results for $p p \rightarrow$ $H+j \rightarrow \gamma \gamma+j$ closely following the ATLAS $8 \mathrm{TeV}$ analysis and for $p p \rightarrow H+j \rightarrow W^{+} W^{-}+j \rightarrow$ $e^{+} \mu^{-} \nu \bar{\nu}+j$ in a CMS-like $13 \mathrm{TeV}$ setup.
\end{abstract}

DOI: $10.1103 /$ PhysRevD.92.074032

PACS numbers: $12.38 . \mathrm{Bx}, 14.80 . \mathrm{Bn}$

\section{INTRODUCTION}

Studies of the Higgs boson discovered by the ATLAS and CMS collaborations [1,2] will be at the focus of the experimental program during Run II of the LHC. The interpretation of future measurements of Higgs boson production and decay rates in terms of Higgs boson couplings to matter and gauge fields and Higgs boson quantum numbers will rely on the comparison of measured event rates and kinematic distributions with results of theoretical modeling of such processes in the Standard Model. It is hoped that such comparisons will help to elucidate the nature of the Higgs particle and explore the mechanism of electroweak symmetry breaking in detail $[3,4]$.

Recently, the ATLAS Collaboration made an important step forward in presenting the results of an analysis of the Higgs boson production and decay at the LHC. Indeed, in contrast to many other Run I LHC measurements, the ATLAS Collaboration measured fiducial volume cross sections and a variety of kinematic distributions in the process $p p \rightarrow H+$ jets [5]. The Higgs boson was identified through its decay to photons, $H \rightarrow \gamma \gamma$.

Fiducial volume measurements allow for a direct comparison between data and theoretical predictions minimizing extrapolation uncertainties. Although many of the ATLAS fiducial volume measurements are currently limited by statistical uncertainties, this will certainly change in the current run of the LHC. Therefore, the important issue for the near future is the availability of highly accurate

\footnotetext{
*fabrizio.caola@cern.ch

†irill.melnikov@kit.edu

*markus.schulze@cern.ch
}

Published by the American Physical Society under the terms of the Creative Commons Attribution 3.0 License. Further distribution of this work must maintain attribution to the author(s) and the published article's title, journal citation, and DOI. theoretical predictions that can be used to describe complicated fiducial volume measurements.

We will now summarize the most advanced fixed order computations related to Higgs boson production and decay at the LHC. The inclusive production cross section of the Higgs boson has recently been computed through next-tonext-to-next-to-leading order in perturbative QCD [6]. This computation refers to the total cross sections and cannot be used for the direct comparison with fiducial volume measurements without extrapolation. The computation of $H+j$ production at the LHC has recently been extended to next-tonext-to-leading order (NNLO) in perturbative QCD, in a fully differential manner $[7,8] .{ }^{1}$ Unfortunately, in Refs. [7,8] decays of the Higgs boson were not considered; for this reason the comparison of the results of Refs. $[7,8]$ with the results of the fiducial measurements is also not possible. When the Higgs boson is produced in association with two and three jets, the NLO QCD computations provide the state-of-the-art results [11-14]. In those NLO QCD computations decays of the Higgs bosons are routinely taken into account. We note that the NNLO QCD computations of $p p \rightarrow H+j[7,8]$ combine the NNLO prediction for the exclusive $H+j$ cross section with the NLO QCD prediction for the exclusive $H+2 j$ cross section and the LO prediction for the $H+3 j$ cross section, making them particularly suitable for studying Higgs boson production in association with a different number of jets in a consistent way. Predictions for Higgs boson decays into a pair of bottom quarks are known through NNLO QCD $[15,16]$. Similarly, the process $H \rightarrow \gamma \gamma$ is known to two loops in QCD and the electroweak model [17-20]. The more complex Higgs boson decays through $Z Z$ and $W W$ bosons into four-lepton final states, which involve finite width and interference effects, are known to NLO [21,22]. Dominant two-loop electroweak

\footnotetext{
${ }^{1}$ For earlier partial results, see $[9,10]$.
} 
corrections in the heavy-Higgs limit have been presented in Refs. [23,24].

It is relatively straightforward to extend the fully differential $p p \rightarrow H+j$ computation reported in Ref. [7] to include decays of the Higgs boson into electroweak gauge bosons since the Higgs boson is a spin-zero particle and no spin correlations need to be considered. This is what we do in this paper for a variety of the Higgs boson decay modes. Once this is done, it becomes possible to calculate fiducial volume cross sections and kinematic distributions and directly compare with experimental measurements. The very fact that it is possible to do that through next-to-nextto-leading order in the expansion in the strong coupling constant, represents an impressive milestone in an application of perturbative QCD to the description of hard collisions at the LHC.

Our paper is organized as follows. In Sec. II, we briefly summarize the theoretical and experimental setup. In Sec. III, we present the results for fiducial volume cross sections and kinematic distributions at the $8 \mathrm{TeV}$ LHC for the $H \rightarrow \gamma \gamma$ decay mode and at the $13 \mathrm{TeV}$ LHC for the $H \rightarrow W W^{*} \rightarrow e^{+} \mu^{-} \nu \bar{\nu}$ decay mode. We also compare the results of the fiducial volume computation for the $\gamma \gamma$ final state with the results of the ATLAS measurement. We conclude in Sec. IV.

\section{THE SETUP}

\section{A. Theory}

We begin by summarizing the theoretical framework that we use in the computation. We work in an effective field theory obtained by integrating out the top quark. We employ the method of improved sector decomposition developed in Refs. [25-27]. This method is based on the factorization of scattering amplitudes in soft and collinear limits and on a particular way of splitting the phase space into sectors, where soft and collinear singularities are easily identified. For this calculation, we require a large number of matrix elements that are used to construct differential cross sections. In particular, we need the two-loop virtual corrections to the partonic channels $g g \rightarrow H g$ and $q g \rightarrow H q$, the one-loop virtual corrections to $g g \rightarrow H g g$, $g g \rightarrow H q \bar{q}, q g \rightarrow H q g, q \bar{q} \rightarrow H Q \bar{Q}$, and the double real emission processes $g g \rightarrow H g g g, g g \rightarrow H g q \bar{q}, q g \rightarrow H q g g$ and $q g \rightarrow H q Q \bar{Q}$, where the $Q \bar{Q}$ pair can be of any flavor. The helicity amplitudes for all of these processes are available in the literature. The two-loop amplitudes were computed in Ref. [28]. The one-loop corrections to the four-parton processes are known [29]. For five-parton treelevel amplitudes, we use compact results obtained using Britto-Cachazo-Feng-Witten recursions [30].

It is nontrivial to combine processes with different particle multiplicities as required for any NNLO QCD computation. Our method for doing that is described in [9]; we do not repeat that discussion here. As already mentioned in the
Introduction, the inclusion of Higgs boson decays is straightforward since the Higgs boson is a spin-zero particle. The only technical issue that arises is a significantly larger phase space that needs to be considered and the ensuing difficulties with the Monte Carlo integration. However, the numerical challenges that appear in fiducial volume computations turn out to be not prohibitive. In particular, we find that for the $H \rightarrow \gamma \gamma$ decay mode we need roughly the same amount of statistics as for the stable Higgs case, while for $H \rightarrow 4 l$ the amount of statistics should be increased by a factor between 2 and 4 .

\section{B. $\boldsymbol{H} \rightarrow \gamma \gamma$}

We continue by listing the selection criteria employed by the ATLAS Collaboration [5]. We are interested in the process $p p \rightarrow H+j$, where the Higgs boson decays to two photons. Final state jets are defined using the anti- $k_{\perp}$ algorithm [31] with $\Delta R=0.4$ and $p_{\perp, j}>30 \mathrm{GeV}$. Jets are required to have rapidities $y_{j}$ in an interval $-4.4<$ $y_{j}<4.4$. The two photons from the Higgs decay must have the transverse momenta $p_{\perp, \gamma_{1}}>\max \left(25 \mathrm{GeV}, 0.35 m_{\gamma \gamma}\right)$ and $p_{\perp, \gamma_{2}}>\max \left(25 \mathrm{GeV}, 0.25 m_{\gamma \gamma}\right)$, respectively, where $m_{\gamma \gamma}$ is the invariant mass of the two photons. In our calculation, the Higgs boson decays are described in the narrow width approximation, so that we always have $m_{\gamma \gamma}=m_{H}=125 \mathrm{GeV}$. Then, the above conditions imply $p_{\perp, \gamma_{1}}>43.75 \mathrm{GeV}$ and $p_{\perp, \gamma_{2}}>31.25 \mathrm{GeV}$. ATLAS requires that the two photons are in the central region of the detector $\left|y_{\gamma}\right|<2.37$, but no photons are allowed to be in the rapidity interval $1.37<\left|y_{\gamma}\right|<1.56$. However, when presenting the results of the measurements [5], the ATLAS Collaboration corrects for the second condition and, for this reason, we do not account for it in our calculation. ${ }^{2}$ It is required that photons and jets are well separated $\Delta R_{\gamma j}>0.4$. For our theoretical predictions we employ parton distribution functions (PDFs) and the strong coupling constant as provided by the NNPDF21LO [32], NNPDF23NLO and NNPDF23NNLO [33] PDF sets to compute respectively LO, NLO and NNLO cross sections. Finally, we take the branching ratio for the Higgs boson decay to two photons to be $\operatorname{Br}(H \rightarrow \gamma \gamma)=2.35 \times 10^{-3}$.

\section{C. $\boldsymbol{H} \rightarrow \boldsymbol{W}^{+} \boldsymbol{W}^{-} \rightarrow \boldsymbol{e}^{+} \boldsymbol{\mu}^{-} \boldsymbol{\nu} \bar{\nu}$}

As the second example, we consider $H+j$ production at the $13 \mathrm{TeV}$ LHC. The Higgs boson decays to $e^{+} \mu^{-} \nu \bar{\nu}$ final state through a pair of $W$ bosons. To identify selection criteria, we apply kinematic cuts similar to those employed by the CMS Collaboration in their studies of the $H \rightarrow W^{+} W^{-}$production at the $8 \mathrm{TeV}$ LHC [34]. We define jets using the anti- $k_{\perp}$ algorithm

\footnotetext{
${ }^{2}$ We note that it is unclear to us why correcting for the missing rapidity region is a worthwhile thing to do since such theoretical correction defies the original goal of comparing theoretical fiducial volume cross sections with the results of experimental measurements.
} 

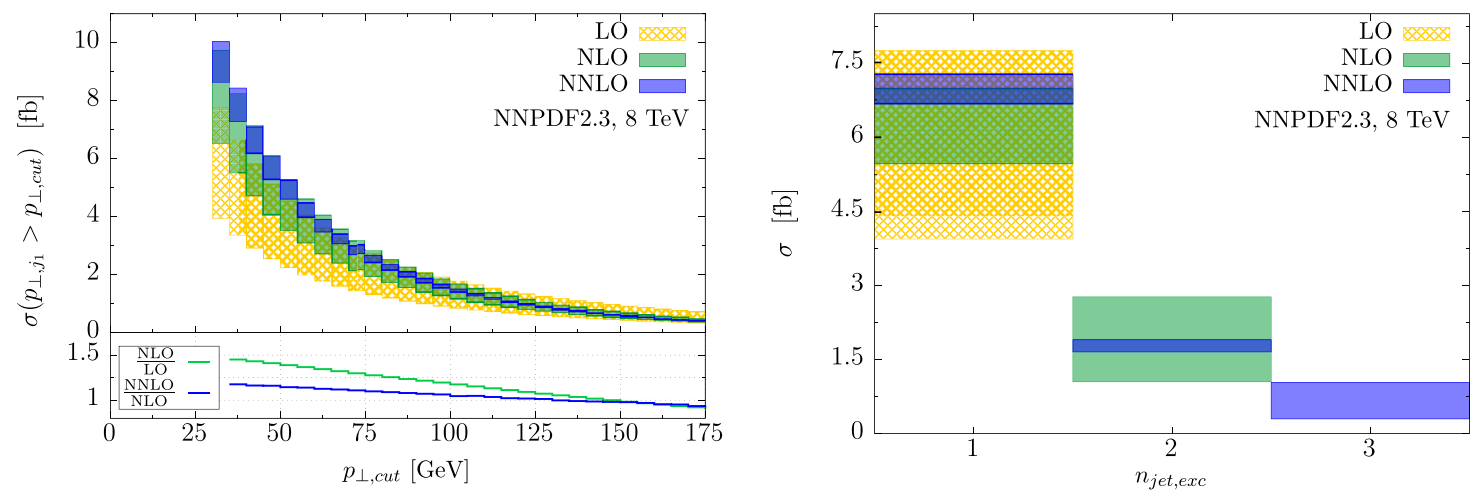

FIG. 1 (color online). Left pane: Fiducial cross section for $p p \rightarrow H(\gamma \gamma)+j$ at the $8 \mathrm{TeV}$ LHC as a function of the cut on the jet transverse momentum. The inset shows ratios of differential cross sections at different orders in perturbation theory for the factorization and the renormalization scales set to the mass of the Higgs boson. Right pane: fiducial cross sections for exclusive jet bins. The selection criteria are described in the text.

with $\Delta R=0.4$. Jets are required to have transverse momentum $p_{\perp, j}>30 \mathrm{GeV}$ and be in the rapidity interval $-4.7<y_{j}<4.7$. The harder of the two charged leptons must have transverse momentum $p_{\perp, l}>20 \mathrm{GeV}$; the softer one must have $p_{\perp, l}>10 \mathrm{GeV}$. The transverse missing energy in the event should exceed $E_{\perp \text {,miss }}>20 \mathrm{GeV}$. Other cuts that we employ are (i) the cut on the dilepton invariant mass $m_{l l}>12 \mathrm{GeV}$, (ii) the cut on the transverse momentum of the dilepton pair $p_{\perp, l l}>30 \mathrm{GeV}$ and (iii) the cut on the transverse mass of the two $W$ bosons $m_{\perp}=$ $\sqrt{2 p_{\perp, l l} E_{\perp, \text { miss }}\left(1-\cos \Delta \phi_{l l, \text { miss }}\right)}>30 \mathrm{GeV}$.

\section{THE RESULTS}

$$
\text { A. } H \rightarrow \gamma \gamma
$$

We consider production of the Higgs boson in association with a jet at the $8 \mathrm{TeV}$ LHC and compute the fiducial volume cross section and the kinematic distributions using the ATLAS selection criteria described in Sec. II. We begin with the fiducial volume cross section. For events that contain the Higgs boson and at least one jet, the ATLAS Collaboration obtains [5]

$$
\begin{aligned}
\sigma_{H+j}^{\mathrm{fid}}(8 \mathrm{TeV})= & 21.5 \pm 5.3(\text { stat }) \pm_{2.2}^{2.4}(\text { syst }) \\
& \pm 0.6 \text { (lumi) fb. }
\end{aligned}
$$

This result is significantly higher than what the fixed order computation predicts. For the $8 \mathrm{TeV}$ LHC we obtain inclusive fiducial $H(\gamma \gamma)+j$ production cross sections ${ }^{3}$

$$
\begin{aligned}
\sigma_{\mathrm{LO}}^{\mathrm{fid}} & =5.43_{-1.49}^{+2.32} \mathrm{fb}, \quad \sigma_{\mathrm{NLO}}^{\mathrm{fid}}=7.98_{-1.46}^{+1.76} \mathrm{fb}, \\
\sigma_{\mathrm{NNLO}}^{\mathrm{fid}} & =9.45_{-0.82}^{+0.58} \mathrm{fb},
\end{aligned}
$$

\footnotetext{
${ }^{3}$ We remind the reader that we work in the $m_{t} \rightarrow \infty$ limit. Moreover, we do not include subleading $q q$ channels beyond NLO; cf. Refs. [7,8].
}

where the central value corresponds to the factorization and renormalization scales set to a common value $\mu=m_{H}$ and the upper (lower) values to $\mu=m_{H} / 2\left(\mu=2 m_{H}\right)$, respectively. Theoretical results indicate reasonable convergence of the perturbative expansion. Indeed, for $\mu=m_{H} / 2$ the NLO cross section is higher than the LO one by $26 \%$, and the NNLO cross section is higher than the NLO one by only $3 \%$. The situation is worse but similar for $\mu=m_{H}$, where the NLO (NNLO) corrections amount to $47 \%$ (18\%), respectively.

It is interesting to point out that the quality of the perturbative expansion for $p p \rightarrow H+j$ appears to be somewhat better than for the inclusive Higgs boson production. As was pointed out earlier in the context of NLO computations, this feature may be related to a cancellation of Sudakov logarithmic corrections $\mathcal{O}\left(\alpha_{s}^{n} \ln ^{2 n} p_{\perp, j} / m_{H}\right)$ that are present in the computation of the $H+j$ cross section, and other sources of large corrections that contribute to the inclusive rate. It was argued that this cancellation is accidental and for this reason cannot be expected to continue in higher orders of perturbation theory. Our calculation questions this assertion. Indeed, it clearly demonstrates that, through the NNLO in perturbative QCD, there is no indication that fixed order perturbation theory for $H+j$ production with the cut on the jet transverse momentum $p_{\perp, j} \geq 30 \mathrm{GeV}$ breaks down.

As a further illustration of this point, we show in the left pane of Fig. 1 the cross sections for the $p p \rightarrow H+j$ process as a function of the jet transverse momentum cut $p_{\perp, \text { cut }}$. It follows from this plot that both the NLO and the NNLO QCD corrections are moderate for all values of $p_{\perp \text {,cut }}$. In particular, the convergence of perturbative series for the cross section with $p_{\perp \text {,cut }}=30 \mathrm{GeV}$ does not appear to be significantly worse than the convergence of perturbative predictions for larger values of the transverse momentum cut.

It is interesting to point out that in Ref. [5] a summary of the theoretical results for $H+j$ fiducial volume cross 

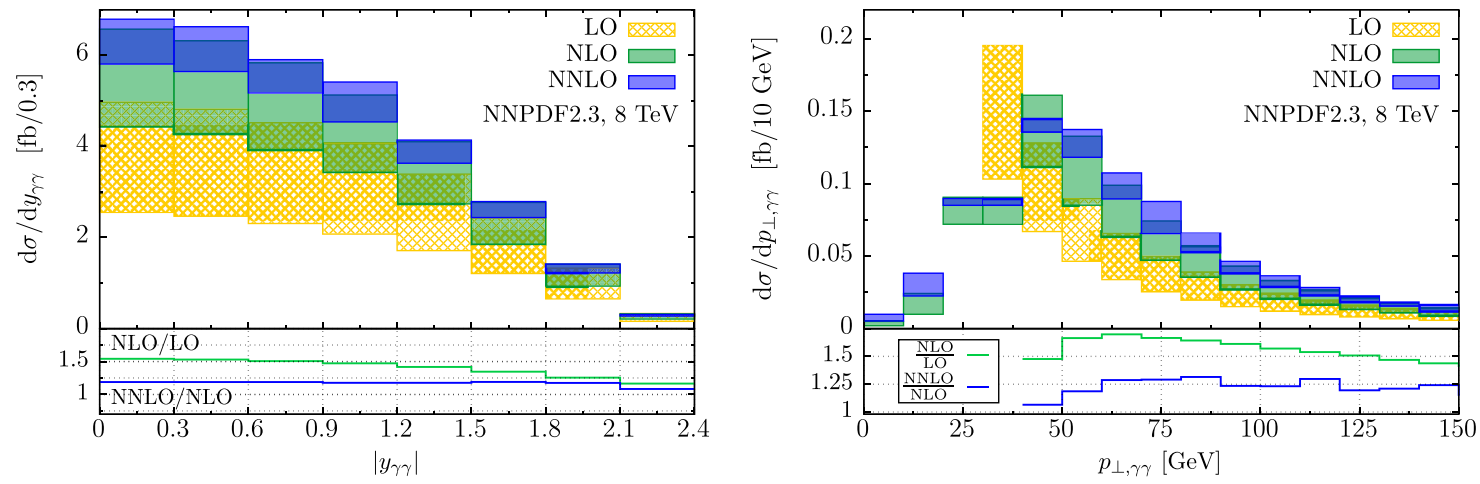

FIG. 2 (color online). Higgs boson rapidity (left) and transverse momentum (right) distributions at the 8 TeV LHC. The insets show ratios of differential cross sections at different orders in perturbation theory for the factorization and the renormalization scales set to the mass of the Higgs boson.

sections was presented, based on exact NLO QCD computations and various resummations of potentially enhanced terms [35-37]. Compared to these three results, our result is somewhat higher than the result of Ref. [35] and it is lower than the two results based on the calculations of Refs. $[36,37] .{ }^{4}$ On the other hand, the uncertainty of our result is significantly smaller.

Nevertheless, the difference between the measured $p p \rightarrow H+j$ cross section by the ATLAS Collaboration and our theoretical prediction is striking - the central value measured by ATLAS is higher than our results by a factor 2.1-2.5, depending on the choice of the renormalization and factorization scale. If one accounts for the uncertainty of the experimental result, this difference translates to, approximately, 2.4 standard deviations and is not statistically significant. On the other hand, it is interesting to remark that the mismatch in the $H+j$ channel is stronger than the mismatch in the inclusive $p p \rightarrow H$ cross section, where the experimental cross section exceeds the theoretical one by a factor of 1.4 [5]. It will be interesting to watch how results of these measurements will evolve in the future especially since, thanks to the availability of the NNLO QCD computation, the precision of theoretical calculations is quite high.

We can recast our results in Eq. (2) into predictions for acceptances, at different orders in perturbation theory. An acceptance is defined as the ratio of a fiducial to total cross section $A=\sigma_{\text {fid }} / \sigma$ for $H+j$ production. When ratios of cross sections are computed, many sources of theoretical uncertainties cancel out and it is in general not possible to properly estimate the uncertainty of the result by changing factorization and renormalization scales within a prescribed interval. For this reason, it is useful to know several orders in the perturbative expansion of the acceptance, to estimate the precision with which it can actually be predicted. For the $8 \mathrm{TeV}$ LHC and the ATLAS setup, we find

\footnotetext{
${ }^{4}$ Note however that finite mass effects can increase the cross section by about $6 \%$ [38-40].
}

$$
\begin{aligned}
A_{\mathrm{LO}} & =0.594(4), \\
A_{\mathrm{NLO}} & =0.614(3), \\
A_{\mathrm{NNLO}} & =0.614(4) .
\end{aligned}
$$

The perturbative expansion for the acceptances exhibits good convergence. Indeed, by comparing the central values, we find that the NLO acceptance is larger than the $\mathrm{LO}$ acceptance by $3 \%$, whereas there is no change going from NLO to NNLO.

Another interesting quantity is the exclusive cross section for a fixed number of jets. The corresponding results are shown in the right pane of Fig. 1. We observe good convergence of the perturbative expansion for the exclusive $H+j$ and $H+2 j$ production cross sections at the $8 \mathrm{TeV}$ LHC. We cannot discuss the perturbative behavior of the $H+3 j$ cross section since it enters our computation only at leading order in perturbative QCD.

We now turn to kinematic distributions studied by the ATLAS Collaboration. They can be divided into three categories: transverse momentum and rapidity distributions of the Higgs boson, transverse momentum, rapidity and the transverse energy distributions of the accompanying QCD radiation and, finally, kinematic distributions of individual photons. The latter includes the transverse momentum and the rapidity distributions as well as the distribution of the photon decay angle in the Collins-Soper reference frame. We can compute all these kinematic distributions through NNLO in perturbative QCD, using exactly the same setup that the ATLAS Collaboration employs in the actual measurement.

We begin with the discussion of the rapidity and the transverse momentum distributions of the Higgs boson in events with at least one jet; see Fig. 2. The pattern of radiative corrections is similar to the fiducial cross section case that we just discussed. In the two plots in Fig. 2 the relative magnitude of radiative corrections is illustrated in lower panes, where ratios of NLO to LO and NNLO to NLO distributions at $\mu=m_{H}$ are displayed. We will refer to such ratios as $K$ factors. We note that similar to the case 

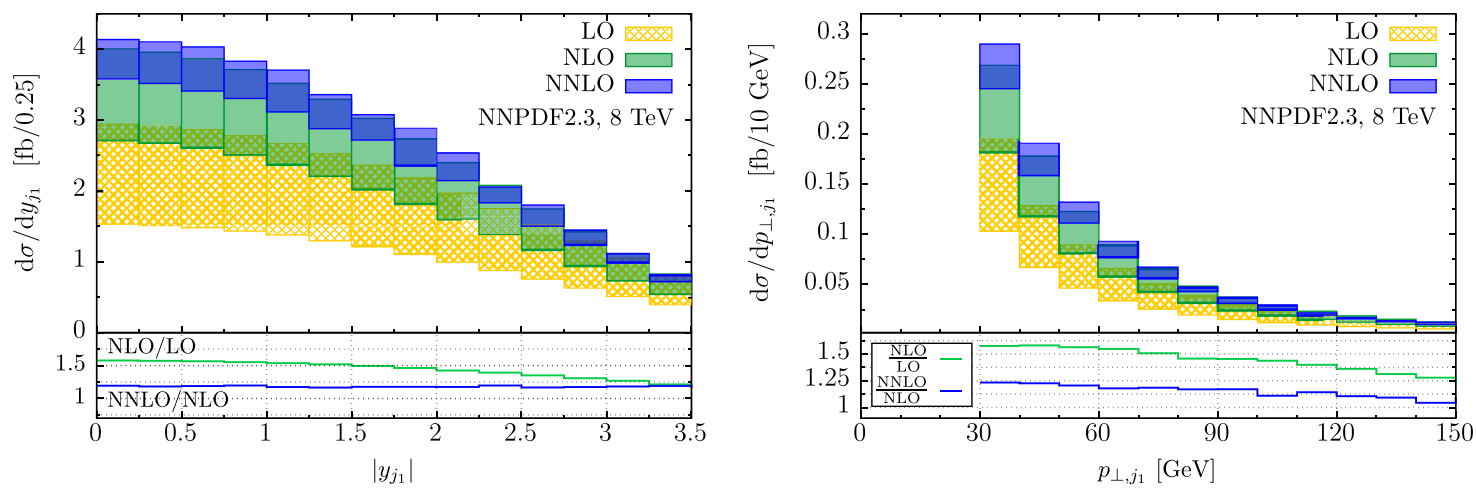

FIG. 3 (color online). Rapidity and transverse momentum distributions of the most energetic jet at the 8 TeV LHC. The insets show ratios of differential cross sections at different orders in perturbation theory for the factorization and the renormalization scales set to the mass of the Higgs boson.

of the inclusive Higgs boson production $p p \rightarrow H$, the NNLO enhancement of the Higgs boson rapidity distribution in the $p p \rightarrow H+j$ process is independent of the rapidity. On the contrary, the $K$ factors for transverse momenta distributions have a more interesting shape. Indeed, we observe the instability of $\mathrm{d} \sigma / \mathrm{d} p_{\perp, H}$ at the value of the Higgs boson transverse momentum equal to the value of the jet transverse momentum cut. This is the manifestation of the so-called Sudakov-shoulder effect [41]. Just above $p_{\perp, H} \sim 30 \mathrm{GeV}$, the NNLO corrections are small but they increase to about $30 \%$ at around $p_{\perp, H} \sim 75 \mathrm{GeV}$ and then start to decrease again.

Next, we consider kinematic distributions of the QCD radiation that accompanies the Higgs boson production. The rapidity and the transverse momentum distributions of the hardest jet are shown in Fig. 3. Similar to the QCD corrections to the Higgs boson rapidity distribution, the NNLO $K$ factor for the hardest jet rapidity distribution is flat. The transverse momentum distribution is reshaped slightly, with corrections being larger at smaller $p_{\perp, j}$ and smaller at larger $p_{\perp, j}$. The distribution of the total transverse energy $H_{\perp}$ of $\mathrm{QCD}$ radiation defined as $H_{\perp}=$ $\sum_{i} p_{\perp, j_{i}}$ is shown in Fig. 4. The sum is taken over all jets observed in a given event. The NNLO QCD corrections for this observable are smaller for smaller $H_{\perp}$, but, eventually, they increase and flatten out. This is similar to what happens to this observable already at next-to-leading order.

Finally, we turn to distributions that describe kinematic properties of individual photons that originate from the Higgs boson decays. In Fig. 5, we show transverse momentum and rapidity distributions of the two photons. Similar to other cases, we find a uniform enhancement of the rapidity distribution and some shape-dependent NNLO effects in transverse momenta distributions. However, the shape dependence of QCD corrections is significantly reduced at NNLO compared to the NLO case. This is particularly true for events with transverse momenta at around maximal values for the transverse momenta of both the harder and the softer photons.
In Fig. 6, we show the distribution of the photon decay angles in the Collins-Soper reference frame defined as

$$
\cos \theta^{*}=\left|\sinh \left(y_{\gamma_{1}}-y_{\gamma_{2}}\right)\right| \frac{2 p_{\perp \gamma_{1}} p_{\perp \gamma_{2}}}{m_{H}^{2} \sqrt{1+\left(p_{\perp, H} / m_{H}\right)^{2}}} .
$$

The $\cos \theta^{*}$ distribution is important for studying the spinparity quantum numbers of the Higgs boson [42]. We find that the shape of this distribution is very well predicted by leading-order perturbative QCD computations, with both NLO and NNLO QCD corrections providing a uniform enhancement. This observation should enable the reduction of the uncertainty associated with the modeling of this observable and, perhaps, lead to improved limits on exotic features of the observed Higgs resonance.

Finally, in Fig. 7 we compare the ATLAS measurements with our computations of the fiducial volume signal in $p p \rightarrow H+j \rightarrow \gamma \gamma+j$. The inclusive one-jet cross section was already discussed at the beginning of this section; we remind the reader that the result of the ATLAS

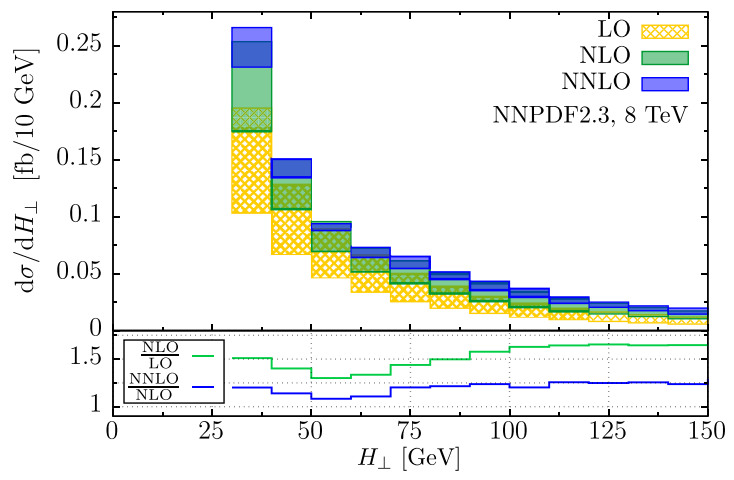

FIG. 4 (color online). The distribution of the total transverse energy $H_{\perp}$ in $H+j \rightarrow \gamma \gamma+j$ production for the $8 \mathrm{TeV}$ LHC. The inset shows ratios of differential cross sections at different orders in perturbation theory for the factorization and the renormalization scales set to the mass of the Higgs boson. 

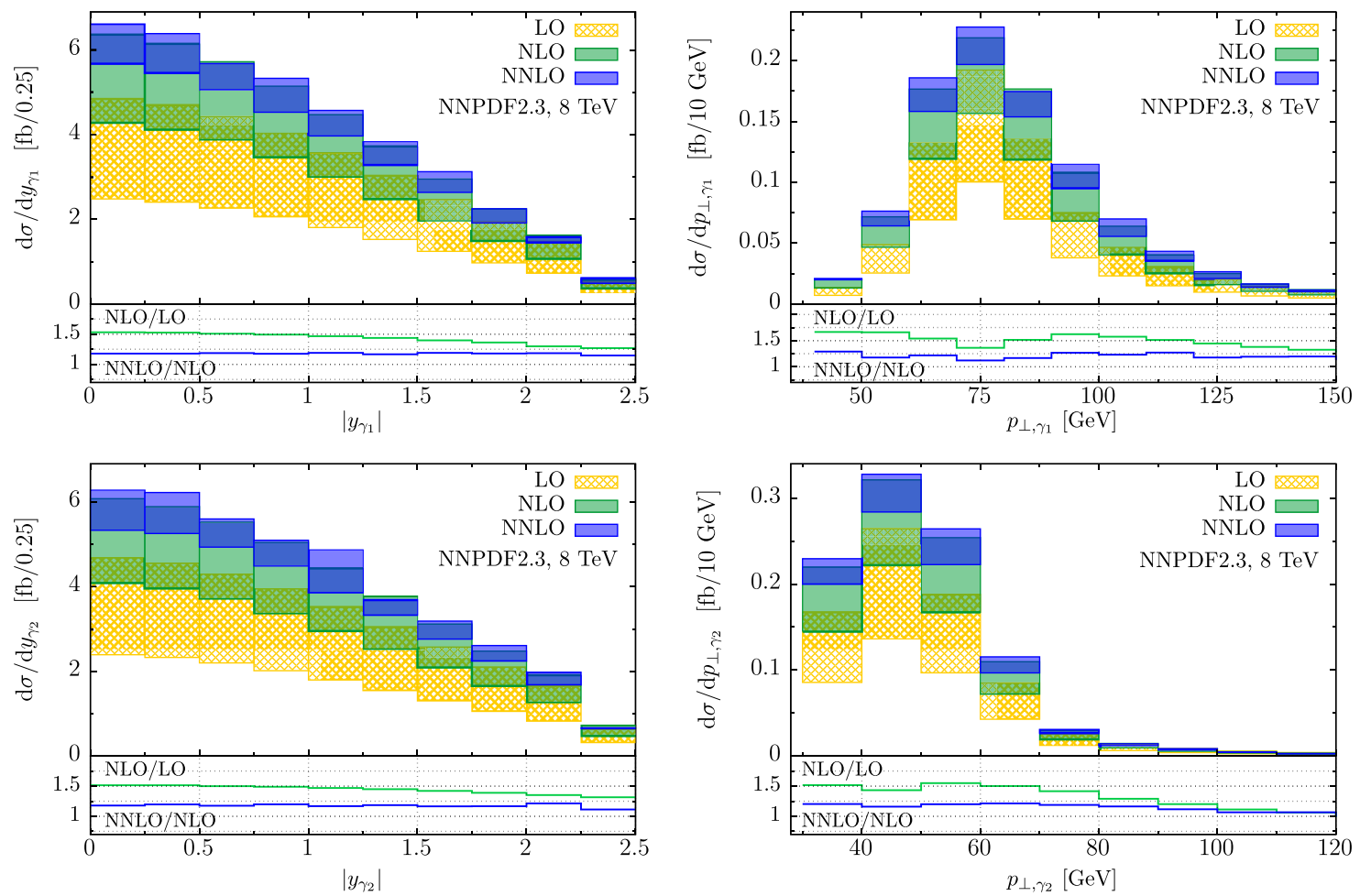

FIG. 5 (color online). Rapidity and transverse momentum distributions of the harder and softer photons in $p p \rightarrow H+j$ at the 8 TeV LHC. The insets show ratios of differential cross sections at different orders in perturbation theory for the factorization and the renormalization scales set to the mass of the Higgs boson.

measurement is significantly higher than the NNLO QCD prediction for the inclusive one-jet cross section. In the left pane of Fig. 7 we present a similar comparison for the exclusive jet cross sections. We see that the situation is similar for all jet multiplicities and that the discrepancy increases for higher-multiplicity bins. In the right pane of Fig. 7, theoretical and experimental results for the transverse momentum distribution of the hardest jet are compared. For this observable, the ATLAS results are higher than the theoretical predictions in all $p_{\perp}$ bins except one where the experimental error is the largest. It is also clear that the shapes of theoretical and experimental distributions are different. It follows from the plots in Fig. 7 that, currently, the ATLAS data are not precise enough to allow for a meaningful comparison with available theoretical predictions. This will undoubtedly change once enough luminosity at the $13 \mathrm{TeV}$ LHC is collected.

\section{B. $\boldsymbol{H} \rightarrow \boldsymbol{W}^{+} \boldsymbol{W}^{-} \rightarrow \boldsymbol{e}^{+} \boldsymbol{\mu}^{-} \boldsymbol{\nu} \bar{\nu}$}

In this subsection, we present the results for the process $p p \rightarrow H+j \rightarrow W^{+} W^{-}+j$ at the $13 \mathrm{TeV}$ LHC. The selection criteria are described in Sec. II. In principle, many kinematic distributions that can be studied in the $H+j$ production process are independent of the decay mode of the Higgs boson. To avoid overlap with the previous subsection, we present here only those distributions that are particular to the $W^{+} W^{-}$final state.
We begin, however, with the discussion of the fiducial cross sections. We find

$$
\begin{aligned}
\sigma_{\mathrm{LO}}^{\mathrm{fid}} & =13.0_{-3.4}^{+5.1} \mathrm{fb}, \quad \sigma_{\mathrm{NLO}}^{\mathrm{fid}}=18.6_{-3.1}^{+3.7} \mathrm{fb}, \\
\sigma_{\mathrm{NNLO}}^{\mathrm{fid}} & =21.9_{-1.7}^{+0.9} \mathrm{fb} .
\end{aligned}
$$

In general, the perturbative expansion of the $13 \mathrm{TeV}$ cross sections is similar to what was observed at $8 \mathrm{TeV}$. At $\mu=m_{H}$, the NLO cross section is larger than the LO cross section by $43 \%$ and the NNLO cross section exceeds the

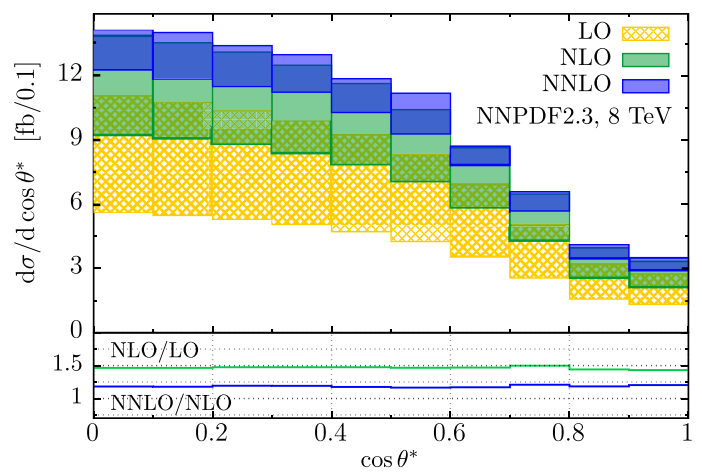

FIG. 6 (color online). Distribution of the photon decay angle in the Collins-Soper frame at the $8 \mathrm{TeV}$ LHC. The inset shows ratios of differential cross sections at different orders in perturbation theory for the factorization and the renormalization scales set to the mass of the Higgs boson. 

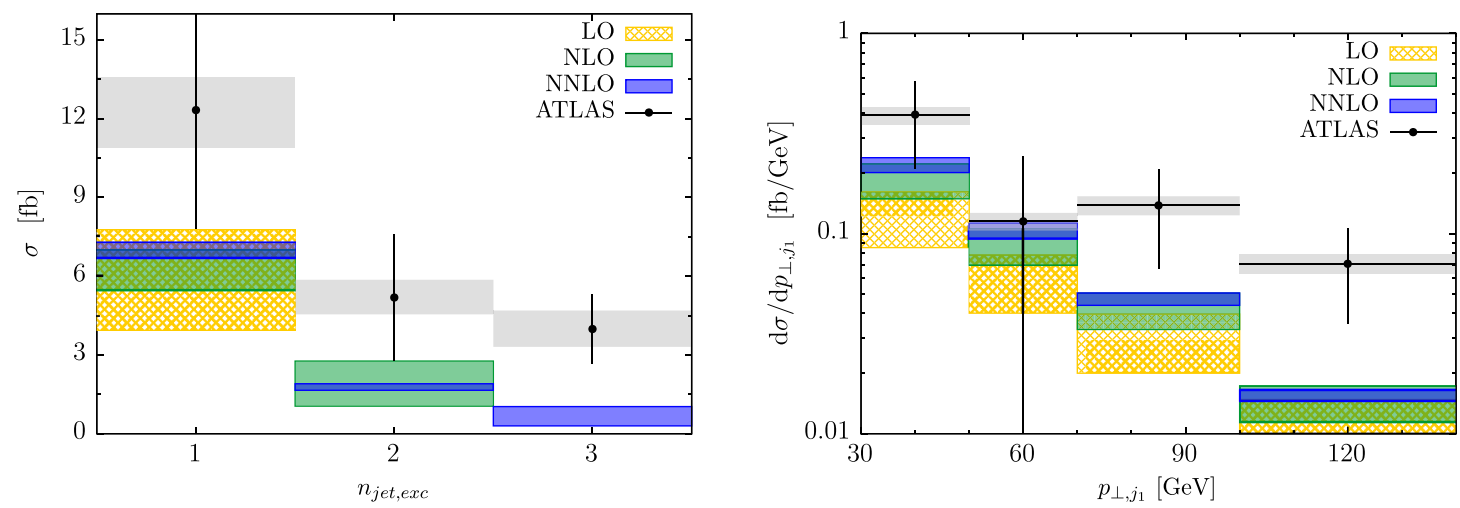

FIG. 7 (color online). Left pane: comparison of exclusive jet cross sections in $p p \rightarrow H+j \rightarrow \gamma \gamma+j$ computed in this paper and measured by the ATLAS Collaboration. Right pane: comparison of the leading jet transverse momentum distribution. The selection criteria are described in the text.
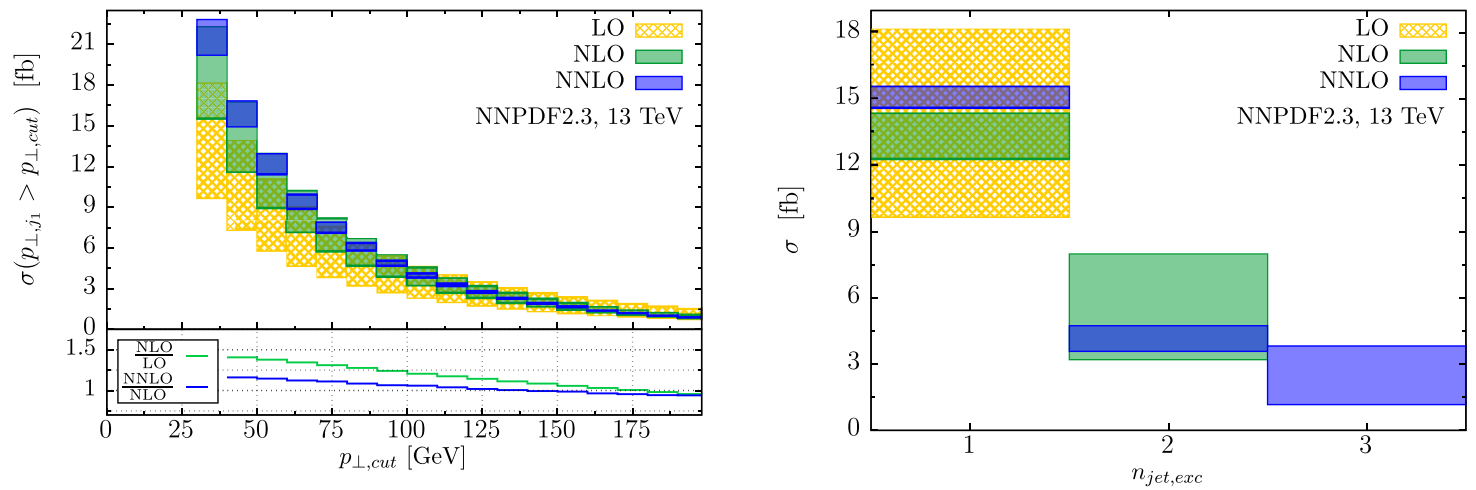

FIG. 8 (color online). Left pane: the production cross section for $p p \rightarrow H+j \rightarrow W^{+} W^{-}+j \rightarrow e^{+} \mu^{-} \nu \bar{\nu}+j$ at the 13 TeV LHC is shown as a function of the jet transverse momentum cut. The inset shows ratios of differential cross sections at different orders in perturbation theory for the factorization and the renormalization scales set to the mass of the Higgs boson. Right pane: exclusive jet cross sections for $p p \rightarrow H\left(e^{+} \mu^{-} \nu \bar{\nu}\right)+j$ at the $13 \mathrm{TeV}$ LHC. The selection criteria are described in the text.
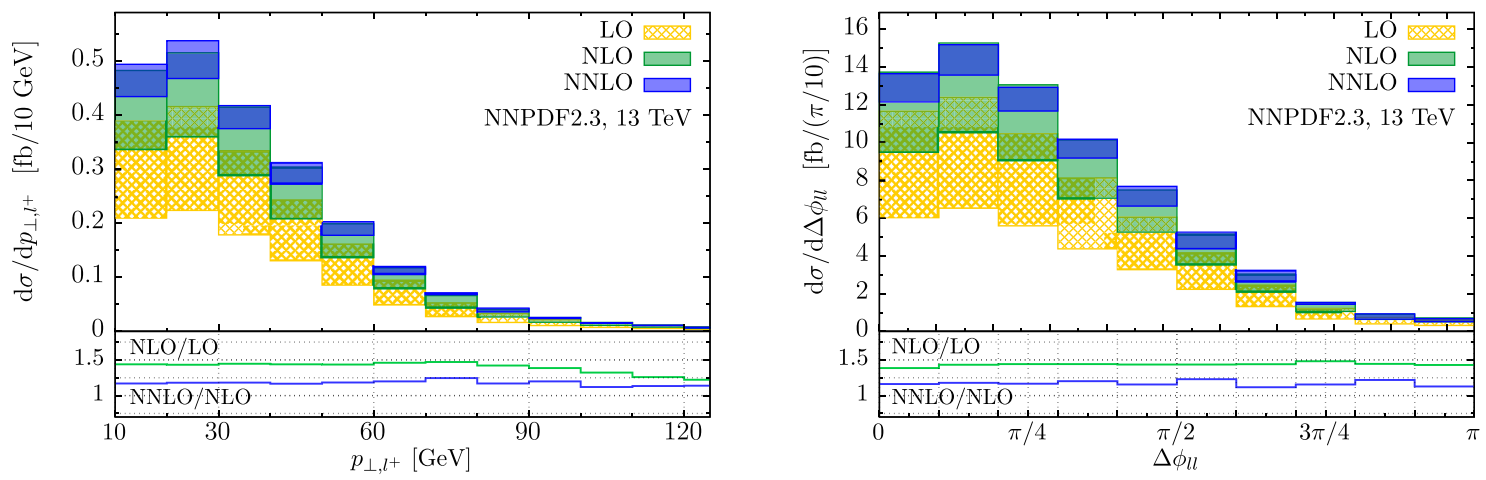

FIG. 9 (color online). Left pane: the transverse momentum distribution of a positively charged lepton in $p p \rightarrow H\left(e^{+} \mu^{-} \nu \bar{\nu}\right)+j$ at the $13 \mathrm{TeV}$ LHC. Right pane: the distribution of the azimuthal opening angle of the two leptons in $p p \rightarrow H\left(e^{+} \mu^{-} \nu \bar{\nu}\right)+j$ at the $13 \mathrm{TeV}$ LHC. The selection criteria are described in the text. The insets show ratios of differential cross sections at different orders in perturbation theory for the factorization and the renormalization scales set to the mass of the Higgs boson.

NLO cross section by $18 \%$. In Fig. 8 we display results for cross sections as a function of the jet transverse momentum cut and exclusive jet cross sections at different orders in perturbation theory. The behavior of the exclusive one-jet cross section at $13 \mathrm{TeV}$ is slightly worse than at $8 \mathrm{TeV}$. We attribute this to a too small scale variation at NLO, which leads to the NNLO result for the one-jet cross section being outside of the NLO scale variation band. 

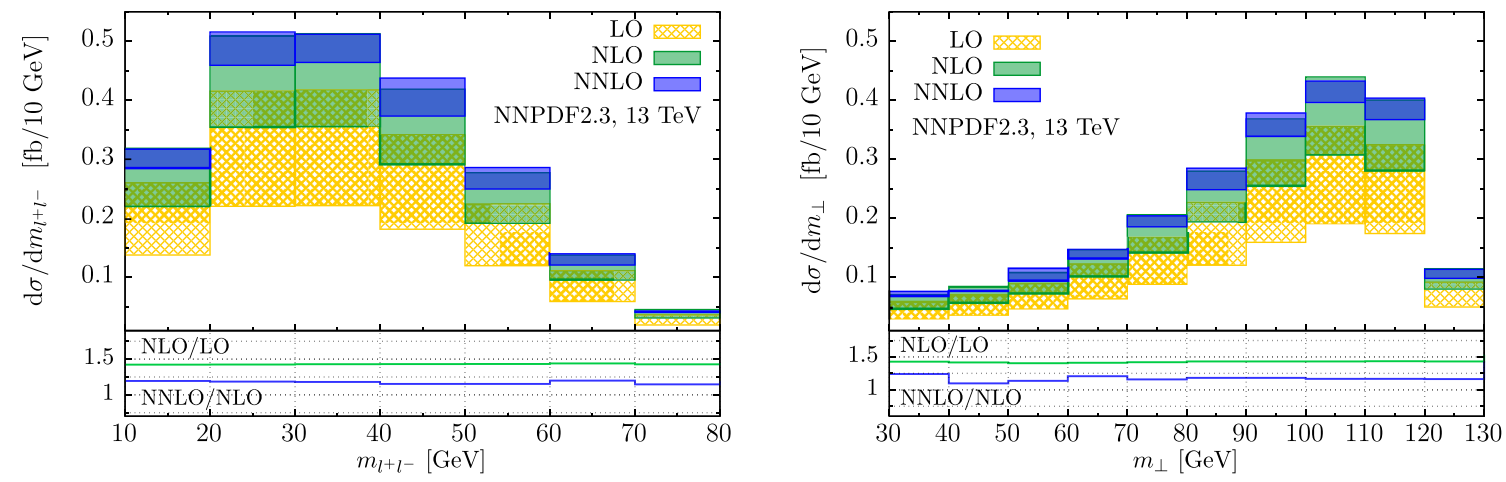

FIG. 10 (color online). Left pane: the lepton invariant mass distribution in $p p \rightarrow H\left(e^{+} \mu^{-} \nu \bar{\nu}\right)+j$ at the 13 TeV LHC. Right pane: the $W^{+} W^{-}$boson transverse mass distribution in $p p \rightarrow H\left(e^{+} \mu^{-} \nu \bar{\nu}\right)+j$ at the $13 \mathrm{TeV}$ LHC. The selection criteria are described in the text. The insets show ratios of differential cross sections at different orders in perturbation theory for the factorization and the renormalization scales set to the mass of the Higgs boson.

Selection criteria for the Higgs signal in $H \rightarrow W^{+} W^{-}$as well as analysis of anomalous couplings in this process rely on kinematic distributions of charged leptons. A good understanding of these distributions is therefore important. In Fig. 9 we show the transverse momentum distribution of a positively charged lepton in $p p \rightarrow H+j \rightarrow W^{+} W^{-}+$ $j \rightarrow e^{+} \mu^{-} \nu \bar{\nu}+j$ at the $13 \mathrm{TeV}$ LHC and the azimuthal opening angle distribution of the two leptons. In both cases, QCD radiative corrections do not change the shapes of the distributions significantly. The distribution of the invariant masses of the two leptons $m_{l^{+} l^{-}}$and the transverse mass $m_{\perp}$ are displayed in Fig. 10; the NNLO QCD corrections to those distributions are remarkably uniform.

\section{CONCLUSIONS}

We extended the recent NNLO QCD computation of the $H+j$ production in proton collisions by including decays of the Higgs bosons to electroweak gauge bosons $H \rightarrow \gamma \gamma$, $H \rightarrow W^{+} W^{-}$and $H \rightarrow Z Z$. Leptonic decays of $Z$ 's and $W$ 's, with all spin correlations, are fully accounted for. This allowed us to calculate fiducial volume cross sections and various kinematic distributions through NNLO in perturbative QCD in a manner that is fully consistent with selection criteria applied in experiments. In particular, it becomes possible-for the first time- to confront fiducial volume studies of the $p p \rightarrow H+j \rightarrow \gamma \gamma+j$ process performed by the ATLAS Collaboration at the $8 \mathrm{TeV}$ LHC [5] with NNLO QCD predictions.

We presented a number of results for fiducial volume cross sections, acceptances and various kinematic distributions for both inclusive and exclusive $H(\gamma \gamma)+j$ production processes. We found no indication that perturbative QCD breaks down and requires resummation for the jet cut as low as $30 \mathrm{GeV}$. We also studied the $W W$ final state in $H+j$ production at the $13 \mathrm{TeV}$ LHC. We found that most of the kinematic distributions used to distinguish this channel from backgrounds show uniform enhancement when NNLO QCD corrections are included. Changes of shapes of such distributions - if any - are already properly captured by the NLO QCD computations.

As a final remark, we note that the availability of higher order QCD predictions for fiducial volume quantities should allow direct and precise studies of the ratios of Higgs signals. The idea that ratios of cross sections are useful for reducing theoretical uncertainties is, of course, well known and appreciated. However, given the availability of the NNLO QCD computations for fiducial cross sections, no extrapolations should be required. As an illustration we computed the ratio of fiducial cross sections for $p p \rightarrow H+j \rightarrow \gamma \gamma+j$ at the $8 \mathrm{TeV}$ LHC and the $p p \rightarrow$ $H+j \rightarrow W W^{*}+j \rightarrow e^{+} \mu^{-} \nu \bar{\nu}+j$ at the $13 \mathrm{TeV}$ LHC. We obtained

$$
\begin{aligned}
R_{W W / \gamma \gamma}= & \frac{\sigma_{H+j}^{W W \rightarrow e^{+} \mu^{-} \nu \bar{\nu}, 13 \mathrm{TeV}}}{\sigma_{H+j}^{\gamma \gamma, 8 \mathrm{TeV}}}=2.39_{+0.04}^{-0.06}, \\
& 2.33_{+0.05}^{-0.04}, \quad 2.32_{+0.02}^{-0.04},
\end{aligned}
$$

at leading, next-to-leading and next-to-next-to-leading order in perturbative QCD, respectively. The convergence of the series is striking; at NNLO QCD, we were able to predict $R_{W W / \gamma \gamma}$ with the precision of better than $2 \%$. Since $R_{W W / \gamma \gamma}$ is proportional to the ratio of the Higgs couplings to two photons and to $W$ bosons, confronting a precise prediction for this observable with results of experimental measurements should allow for stringent constraints on the deviations of these couplings from their Standard Model values.

\section{ACKNOWLEDGMENTS}

We are grateful to Joey Huston for the clarification of some details of the ATLAS analysis. We thank the Mainz Institute for Theoretical Physics (MITP) for hospitality and partial support during the program Higher Orders and Jets for LHC. 
[1] G. Aad et al. (ATLAS Collaboration), Phys. Lett. B 716, 1 (2012).

[2] S. Chatrchyan et al. (CMS Collaboration), Phys. Lett. B 716, 30 (2012).

[3] G. Aad et al. (ATLAS Collaboration), Report No. ATLASCONF-2013-034, 2013.

[4] S. Chatrchyan et al. (CMS Collaboration), Report No. CMS-PAS-HIG-13-005, 2013.

[5] G. Aad et al. (ATLAS Collaboration), J. High Energy Phys. 09 (2014) 112.

[6] C. Anastasiou, C. Duhr, F. Dulat, F. Herzog, and B. Mistlberger, Phys. Rev. Lett. 114, 212001 (2015).

[7] R. Boughezal, F. Caola, K. Melnikov, F. Petriello, and M. Schulze, Phys. Rev. Lett. 115, 082003 (2015).

[8] R. Boughezal, C. Focke, W. Giele, X. Liu, and F. Petriello, Phys. Lett. B 748, 5 (2015).

[9] R. Boughezal, F. Caola, K. Melnikov, F. Petriello, and M. Schulze, J. High Energy Phys. 06 (2013) 072.

[10] X. Chen, T. Gehrmann, E. W. N. Glover, and M. Jaquier, Phys. Lett. B 740, 147 (2015).

[11] J. M. Campbell, R. K. Ellis, and C. Williams, Phys. Rev. D 81, 074023 (2010).

[12] H. van Deurzen, N. Greiner, G. Luisoni, P. Mastrolia, E. Mirabella, G. Ossola, T. Peraro, J. F. von Soden-Fraunhofen, and F. Tramontano, Phys. Lett. B 721, 74 (2013).

[13] G. Cullen, H. van Deurzen, N. Greiner, G. Luisoni, P. Mastrolia, E. Mirabella, G. Ossola, T. Peraro, and F. Tramontano, Phys. Rev. Lett. 111, 131801 (2013).

[14] N. Greiner, S. Hoeche, G. Luisoni, M. Schonherr, J. C. Winter, and V. Yundin, arXiv:1506.01016.

[15] C. Anastasiou, F. Herzog, and A. Lazopoulos, J. High Energy Phys. 03 (2012) 035.

[16] V. Del Duca, C. Duhr, G. Somogyi, F. Tramontano, and Z. Trcsnyi, J. High Energy Phys. 04 (2015) 036.

[17] U. Aglietti, R. Bonciani, G. Degrassi, and A. Vicini, Phys. Lett. B 600, 57 (2004).

[18] U. Aglietti, R. Bonciani, G. Degrassi, and A. Vicini, Phys. Lett. B 595, 432 (2004).

[19] G. Degrassi and F. Maltoni, Nucl. Phys. B724, 183 (2005).

[20] S. Actis, G. Passarino, C. Sturm, and S. Uccirati, Nucl. Phys. B811, 182 (2009).

[21] A. Bredenstein, A. Denner, S. Dittmaier, and M. M. Weber, J. High Energy Phys. 02 (2007) 080.

[22] A. Bredenstein, A. Denner, S. Dittmaier, and M. M. Weber, Phys. Rev. D 74, 013004 (2006).

[23] A. Ghinculov, Nucl. Phys. B455, 21 (1995).
[24] A. Frink, B. A. Kniehl, D. Kreimer, and K. Riesselmann, Phys. Rev. D 54, 4548 (1996).

[25] M. Czakon, Phys. Lett. B 693, 259 (2010).

[26] M. Czakon and D. Heymes, Nucl. Phys. B890, 152 (2015).

[27] R. Boughezal, K. Melnikov, and F. Petriello, Phys. Rev. D 85, 034025 (2012).

[28] T. Gehrmann, M. Jaquier, E. W. N. Glover, and A. Koukoutsakis, J. High Energy Phys. 02 (2012) 056.

[29] C. F. Berger, V. Del Duca, and L. J. Dixon, Phys. Rev. D 74, 094021 (2006); 76, 099901(E) (2007); S. D. Badger and E. W. N. Glover, Nucl. Phys. B, Proc. Suppl. 160, 71 (2006); S. D. Badger, E. W. N. Glover, and K. Risager, J. High Energy Phys. 07 (2007) 066; E. W. N. Glover, P. Mastrolia, and C. Williams, J. High Energy Phys. 08 (2008) 017; S. D. Badger, E. W. N. Glover, P. Mastrolia, and C. Williams, J. High Energy Phys. 01 (2010) 036; L. J. Dixon and Y. Sofianatos, J. High Energy Phys. 08 (2009) 058; S. Badger, J. M. Campbell, R. K. Ellis, and C. Williams, J. High Energy Phys. 12 (2009) 035.

[30] S. D. Badger (unpublished).

[31] M. Cacciari, G. P. Salam, and G. Soyez, J. High Energy Phys. 04 (2008) 063.

[32] R. D. Ball, V. Bertone, F. Cerutti, L. Del Debbio, S. Forte, A. Guffanti, J. I. Latorre, J. Rojo, and M. Ubiali, Nucl. Phys. B849, 296 (2011).

[33] R. D. Ball, V. Bertone, S. Carrazza, C. S. Deans, L. Del Debbio, S. Forte, A. Guffanti, N. P. Hartland et al., Nucl. Phys. B867, 244 (2013).

[34] S. Chatrchyan et al. (CMS Collaboration), J. High Energy Phys. 01 (2014) 096.

[35] K. Hamilton, P. Nason, and G. Zanderighi, J. High Energy Phys. 10 (2012) 155.

[36] R. Boughezal, X. Liu, F. Petriello, F. J. Tackmann, and J. R. Walsh, Phys. Rev. D 89, 074044 (2014).

[37] A. Banfi, P. F. Monni, G. P. Salam, and G. Zanderighi, Phys. Rev. Lett. 109, 202001 (2012).

[38] E. Bagnaschi, G. Degrassi, P. Slavich, and A. Vicini, J. High Energy Phys. 02 (2012) 088.

[39] M. Grazzini and H. Sargsyan, J. High Energy Phys. 09 (2013) 129.

[40] A. Banfi, P. F. Monni, and G. Zanderighi, J. High Energy Phys. 01 (2014) 097.

[41] S. Catani and B. R. Webber, J. High Energy Phys. 10 (1997) 005 .

[42] G. Aad et al. (ATLAS Collaboration), Phys. Lett. B 726, 120 (2013). 